\title{
Constant Stress Drop from Small to Great Earthquakes in Magnitude- Area Scaling
}

\author{
Bruce E. Shaw \\ Lamont Doherty Earth Observatory, Columbia University
}

\begin{abstract}
Earthquakes span a tremendous range of scales, more than 5 orders of magnitude in length. Are earthquakes fundamentally the same across this huge range of scales, or are the great earthquakes somehow different from the small ones? We show that a robust scaling law seen in small earthquakes, with stress drops being independent of earthquake size, indeed holds for great earthquakes as well. The simplest hypothesis, that earthquake stress drops are constant from the smallest to the largest events, combined with a more thorough treatment of the geometrical effects of the finite seismogenic layer depth, gives a new magnitude area scaling which matches the data well, and better over the whole magnitude range than the currently used scaling laws which have nonconstant stress drop scaling. This has significant implications for earthquake physics and for seismic hazard estimates.
\end{abstract}

\section{Introduction}

How the slip in an earthquake scales with rupture length, or, alternatively, how the magnitude scales with rupture area, has profound implications for earthquake physics, and also, it turns out, seismic hazard estimates. For small earthquakes, "constant stress drop scaling" has been robustly observed [Aki, 1972; Hanks, 1977], whereby slip scales linearly with rupture length, or equivalently magnitude scales linearly with the log of rupture area. What happens for large earthquakes, when the rupture length is large compared to the depth of the seismogenic layer, has been a source of much debate and controversy [Scholz, 1982; Romanowicz, 1992, 1994; Scholz, 1994; Wells and Coppersmith, 1994; Bodin and Brune, 1996; Pegler and Das, 1996; Shaw and Scholz, 2001; Henry and Das, 2001; Hanks and Bakun, 2002]. The leading magnitude-area scaling relations [WGCEP, 2003; Hanks and Bakun, 2002], used in the most recent US national seismic hazard maps, all assume a breakdown of the scaling seen in small earthquake, with stress drops increasing for the largest earthquakes. This poses a huge challenge for earthquake physics and for seismic hazard estimates. What is different in the physics of great earthquakes as compared to their smaller brethren? Even more problematically from the point of view of seismic hazard estimates, how do we use and extrapolate from the much more numerous moderate and destructive large earthquakes to the rare and devastating great earthquakes if the physics differs? With a gaping sparsity of direct measurements for the great earthquakes, yet an essential need to design for their consequences, extrapolating from measurements of more numerous smaller events remains a necessary task. In this paper we show that the simplest hypothesis, that earthquake stress drops are constant from the smallest to the largest events, when combined with a more thorough treatment of the geometrical effects of the finite seismogenic layer depth, gives a magnitude area scaling which matches the data well, and better over the whole magnitude range than the currently used scaling laws which have non-constant stress drop scaling.

The two leading magnitude-area scaling laws, given equal $50 \%$ weighting in the 2007 US National Seismic Hazard maps, both imply increasing stress drops for the largest earthquakes. The so-called "Ellsworth B" model [WGCEP, 2003] is the simplest parameterization, extrapolating the constant relationship between moment magnitude $\mathbf{M}$ [Hanks and Kanamori, 1979] with the log of the rupture area $A$ to the largest events (though with a different offset than is generally used for smaller events 
[Wells and Coppersmith, 1994]):

$$
\mathbf{M}=\log A+4.2
$$

For an infinitely long rupture with a finite width $W$, and a constant stress drop, slip should scale as $W$, so moment scales with area $A$ as $A W$, so moment magnitude which is $2 / 3$ of the log of the moment should scale with $A$ as $\frac{2}{3} \log A$ for fixed $W$. Then to get the $\log A$ magnitude area relationship in Equation (1) for fixed $W$ we would need stress drop $\Delta \sigma$ to scale as $\Delta \sigma \sim A^{1 / 2}$. The second scaling relation, the Hanks-Bakun relation [Hanks and Bakun, 2002], used a bilinear scaling relationship of:

$$
\mathbf{M}= \begin{cases}\log A+3.98 & A \leq 557 \mathrm{~km}^{2} \\ \frac{4}{3} \log A+3.07 & A>557 \mathrm{~km}^{2}\end{cases}
$$

Based on a scaling of slip continuing to increase linearly with rupture length $L$, the so-called "L-model" [Scholz, 1982], this gives an even stronger stress drop scaling for the largest events, with $\Delta \sigma \sim A$.

Our new scaling builds on the $\Delta \sigma \sim A^{0}$ scaling seen for more than five orders of magnitude of $A$ in small earthquakes, by assuming this continues to hold for large earthquakes. We then combine this with finite rupture length $L$ and width $W$ effects, and their interaction with the seismogenic depth $H$ and the free surface. We build our new magnitude area scaling relations by looking at how average slip $D$ scales with rupture dimensions assuming stress drop is constant. Slip times stiffness is stress, and taking for stiffness the inverse lengths in the vertical and horizontal directions of the rupture, Shaw and Scholz [2001] obtained

$$
D \sim \begin{cases}\frac{1}{\frac{1}{L}+\frac{1}{L}} & L \leq 2 W \\ \frac{1}{\frac{1}{L}+\frac{1}{2 W}} & L>2 W .\end{cases}
$$

with the factor of 2 coming from the free surface. Shaw and Scholz [2001] presumed that rupture width $W$ equaled seismogenic depth $H$, and noted the discrepancy of the observed data with the expected scaling, although their dynamic model which had a scale invariant physics matched the observations well. Manighetti et al. [2007] examined the fit of this scaling to the data when $W$ was generalized as a fitting parameter. We can write this as:

$$
D \sim \begin{cases}\frac{1}{\frac{1}{L}+\frac{1}{L}} & L \leq 2 \xi H \\ \frac{1}{\frac{1}{L}+\frac{1}{2 \xi H}} & L>2 \xi H .\end{cases}
$$

Fitting the data for a single best value of $\xi$, Manighetti et al. [2007] found $\xi=3$, though they argued that using multiple values of $\xi$ were a better match and argued for this fitting based on fault segmentation, and not based on fault width. We propose an alternative interpretation of $\xi$ as an effective width. But what might be the cause of such a large effective width? It has generally been presumed that large earthquakes break coseismically only down to the seismogenic depth $H$, but theoretical work is calling this assumption into question. Developing an idea originally proposed by Das [1982], King and Wesnousky [2007] recently showed that if one relaxed the assumption that large earthquakes broke only down to the bottom of the seismogenic layer, and instead kinematically had ruptures penetrating deeper into the stably sliding lower fault, constant stress drop scaling could be maintained while matching observed slip-length scaling relations for large strike-slip earthquakes. Shaw and Wesnousky [2008] went further and showed that three dimensional scalar elastodynamic models which had previously been seen to match slip-length scaling observations [Shaw and Scholz, 2001] indeed dynamically chose this deep penetrating slip behavior. They found that while $W$ increased 
systematically with $L$, a fit as in Equation (4) with a single value $\xi=3$ was a good fit to the data. Significantly, this effective single $\xi$ fit was larger than any individual values of $W / H$ - the increase of $W / H$ with $L$ mapped onto a larger effective single $\xi$. Based on this work, we treat this as a fitting parameter to be compared with the data, and now convert to magnitude-area scaling. Before proceeding, we note that current observations provide insufficient constraints on this question of the depth-of-coseismic slip. King and Wesnousky [2007] discuss in particular the lack of constraints from GPS data due to poor depth resolution; seismic inversions as well are poorly constrained in depth. In short, while substantial coseismic slip below the seismogenic layer has not been directly observed, it has also not been ruled out by observations.

Converting to moment-area scaling, we continue to examine only the seismogenic rupture lengths and area, since the actual downdip widths $W$ of ruptures remain difficult to observe seismologically, whereas the seismogenic depth $H$ can be constrained by seismicity [Nazareth and Hauksson, 2004]. Then, for seismogenic area $A$, and moment $\mathcal{M}$ for now just that associated with that area,

$$
\mathcal{M} \sim A D \sim \frac{A}{\frac{1}{L}+\max \left(\frac{1}{L}, \frac{1}{\beta H}\right)}=\frac{A L}{1+\max \left(1, \frac{L}{\beta H}\right)}
$$

where we have used a new scaling parameter $\beta \equiv 2 \xi$, and we have also combined the two different scalings depending on $L$ relative to $W$ into one expression using the max functional. Note that by "seismogenic area" $A$, we mean the area within the seismogenic width $H$, and do not include any area in $W$ below the seismogenic depth $H$ which may nevertheless rupture coseismically. We consider only the seismogenic area since this is observable, whereas any deeper area which may slip is not unambiguously imageable with current methods. In this way, we make direct contact as well with current seismic hazard estimates which use only seismogenic areas. Translating equation (5) into magnitude $\mathbf{M}$, and writing this as a correction to $\log A$ scaling, we have

$$
\mathbf{M}=\frac{2}{3} \log _{10} \mathcal{M}=\log _{10} A+\frac{2}{3} \log _{10} \frac{L / A^{1 / 2}}{\left(1+\max \left(1, \frac{L}{\beta H}\right)\right) / 2}+\text { const }
$$

Defining a new parameter to be the aspect ratio $\lambda \equiv L / H$ gives the scaling relation

$$
\mathbf{M}=\log _{10} A+\frac{2}{3} \log _{10} \frac{\lambda^{1 / 2}}{(1+\max (1, \lambda / \beta)) / 2}+\text { const }
$$

or, written just in terms of area $A$, and noting $\lambda=1$ for small events, we can write the scaling relations as

$$
\mathbf{M}=\log _{10} A+\frac{2}{3} \log _{10} \frac{\max \left(1, \sqrt{\frac{A}{H^{2}}}\right)}{\left(1+\max \left(1, \frac{A}{H^{2} \beta}\right)\right) / 2}+\text { const } .
$$

Thus far we have only considered the moment from the seismogenic layer. If there is rapid coseismic slip below the seismogenic depth $H$, there will be additional moment coming from this deeper slip. Dynamic modeling studies suggest such slip may occur, but also that the character of the slip may be somewhat different from slip in the seismogenic layer, being much more low frequency in nature and depleted in high frequency [Shaw and Wesnousky, 2008]. From the point of view of moment, however, only the low frequency coseismic motion matters. We can write the additional contribution as

$$
\mathbf{M}=\log _{10} A+\frac{2}{3} \log _{10} \frac{\max \left(1, \sqrt{\frac{A}{H^{2}}}\right)}{\left(1+\max \left(1, \frac{A}{H^{2} \beta}\right)\right) / 2}\left(1+\frac{\mathcal{M}_{z>H}}{\mathcal{M}_{z \leq H}}\right)+\text { const }
$$


where $\mathcal{M}_{z>H}$ is the moment from slip at depths $z$ greater than the seismogenic depth $H$, and $\mathcal{M}_{z \leq H}$ is the seismogenic depth moment we have been considering up to this point. Further parameterizations of the ratio $\mathcal{M}_{z>H} / \mathcal{M}_{z \leq H}$ enable more detailed transitions from the small event regime where $\mathcal{M}_{z>H} / \mathcal{M}_{z \leq H} \approx 0$ to the great event regime where $\mathcal{M}_{z>H} / \mathcal{M}_{z \leq H}$ approaches a constant. Such behavior was seen in dynamic models [Shaw and Wesnousky, 2008]. We have explored a few such parameterizations of the ratio, but have found that the data limitations- the scatter and the limited number of data points and the limited magnitude range over which the ratio changes much- make the additional parameters needed insufficiently distinguishable and not statistically significant in terms of added information content. Instead, we find it most useful to simply absorb this additional ratio into the $\beta$ parameter, so that $\beta$ now encompases both the additional slip in the seismogenic layer due to increased $W$, and the additional moment below the seismogenic layer. In this way, we maintain a minimal parameterization which connect the small event scaling where $\mathbf{M} \sim \log A$ and the great event scaling where $\mathbf{M} \sim 2 / 3 \log A W \sim 2 / 3 \log A H \beta$, and sacrifice some potential details of the interpolating large events connecting the two regimes which are unresolvable with the data. Thus we return back to equation (8) as our proposed scaling for magnitude versus seismogenic area, but with a generalized interpretation of the parameter $\beta$. Equation (8) generalizes the Wells and Coppersmith [1994] and Ellsworth-B [WGCEP, 2003] relations with two additional parameters $\beta$ and $H$. The value of the constant term is set by fitting at the small events. If we assume we know $H$ through other means (e.g. hypocentral depths) then we can fix $H$, and we would have only one additional parameter; otherwise we can set this by fitting to the data. The parameter $\beta$ is set by fitting the data.

\section{Data Fitting}

Because the most revealing data are for large aspect ratio $L / W$ events, we examine strike-slip earthquakes, which have the largest aspect ratios of any mechanism earthquakes. The magnitude-area data are from Hanks and Bakun [2008]. The Hanks and Bakun [2008] data (and other previously used data sets [Wells and Coppersmith, 1994; WGCEP, 2003; Hanks and Bakun, 2002]) do not have error bars, so we assume errors in all data points are the same. Doing a least squares fitting and assuming errors in $\log A$ are the same size as errors in magnitude, we find a best fitting of Equation (8) for parameter values $H=15.6 \mathrm{~km}$ and $\beta=6.9$ to the complete data set.

It is satisfying that the best fitting value of $H$ is so close to generally presumed default worldwide values of $H$. This validates in a fundamental way the physical foundation of our scaling. There is, though, a very broad minimum around this best fitting value, and the constraints on $H$ and $\beta$ are not tight; using the likelihood function and assuming gaussian errors, a one standard deviation contour in the parameters gives bands of $H=15.6_{-3}^{+5} \mathrm{~km}$ and $\beta=6.9_{-1.5}^{+3}$. Assuming the errors in $\log A$ are somewhat larger or somewhat smaller than the errors in $\mathbf{M}$ changes slightly as well the best fitting $H$ and $\beta$ parameters, though their product $H \beta$ remains nearly constant. The more robust metric of absolute value of the distance misfit, as opposed to the square distance, gives a best fitting $H=16.0 \mathrm{~km}$ and $\beta=5.9$.

The fit of the scaling laws to the data are shown in Figure 1. The data, shown with red circles, is seen to be very well fit by the new scaling law, shown with a solid blue line. The leading other scaling relations are also plotted: Wells and Coppersmith [1994] with a solid yellow line, Ellsworth-B [WGCEP , 2003] with a solid black line, and Hanks and Bakun [2002] with a dashed green line.

The new scaling relation appears an excellent fit by eye, and indeed is measured to have a smaller standard deviation than the fits to the other curves $[.1418, .1456, .1927$, for equations (8), (2), and (1), respectively]. It does, however, have more parameters, and thus must beat them sufficiently to make up for that. Using AIC [Akaike, 1974] to penalize for the extra parameters, we find the new scaling is 
nevertheless still better [-90.2, -87.5, -39.0 for Equations (8), (2), and (1), respectively]. An alternative measure of fitting, the Fischer $\mathrm{F}$ test also shows the new scaling to be superior at the $5 \%$ significance level $[F(1,86)=4.65$ for equation (8) relative to equation (2) and $F(2,86)=36.43$ for equation (8) relative to equation (1)]. From a hazard point of view the larger events are more important, and some of the scaling laws have been developed to fit just that region (e.g. Ellsworth-B [WGCEP, 2003]); for completeness we have therefore examined the scaling relations as we fit only above a magnitude threshold, and examine the relative fit as the magnitude threshold is increased. With loss of data points and the narrowing of the range over which the scalings differ, the differences in the fits for just the large events are, however, within the one sigma contour in likelihood, and thus not significant. They therefore do not exceed a significance test which penalizes extra parameters if we restrict the magnitude range to be only above $\mathbf{M} 6.5$ for which equation (1) was developed. Since current seismic hazard analysis considers events down to magnitude 5.5, however, a lower cutoff magnitude is needed. The prospects for more data helping reduce uncertainties are most promising at the lower magnitudes, where modern relative relocation techniques (e.g. [Waldhauser and Ellsworth, 2000]) could be brought to bear, and many well recorded events which have occurred have not been analyzed for inclusion in the data set.

The relatively large value of $\beta$ found has a few origins. First, dynamic models [Shaw and Wesnousky, 2008] fitting the slip-length scaling, Equation (4), found larger single best fitting values when there was some $L$ dependence to $W$. Second, we use the seismogenic area for $A$ in Equation (5) because seismic hazard estimates use this area, because area estimates typically presume this area, and because seismogenic $H$ is much better defined than the difficult to measure $W$. Additional moment and area below the seismogenic zone will map onto our effective $\beta$ value. That is, for very large $A$, $\mathbf{M} \sim 2 / 3 \log A+2 / 3 \log H \beta$ so underestimates in $A$ get mapped onto larger $\beta$ values if $H$ is constrained by the initial increase in magnitude in transitioning from small to large events.

\section{Discussion}

Our new scaling relations have interesting connections with prior work. We get $\log A$ scaling at small magnitudes $\left(A / H^{2}<1\right)$ as all the scaling relations have, " $L$ " scaling of $4 / 3 \log A$ for moderately large events $\left(1<A / H^{2}<\beta\right)$ as Hanks and Bakun [2002] found, and finally a new regime of " $W$ " scaling of $2 / 3 \log A$ for the very largest events $\left(\beta \ll A / H^{2}\right)$.

Our scaling is based on seismogenic depth $H$ being fixed. Observations of seismicity along major faults suggest, however, it can vary along-strike [Nazareth and Hauksson, 2004]. To test the generality of our scaling relation, we have extended our analysis to include a seismogenic depth varying alongstrike $H(x)=H_{0}+\epsilon(x)$ and examined the impact of varying $H(x)$ in the scaling relations. To first order perturbation theory, for $\epsilon(x) / H_{0} \ll 1$, scaling $H_{0}$ so $<\epsilon(x)>_{x}=0$ we find that we can replace $H$ in the scaling relation by its average $H_{0}=<H>_{x}$. This shows a robustness of the results, and extends the utility of our relation.

The maximum deviation of our relation from $\log A$ scaling, occuring at $A=H^{2} \beta$ (ignoring magnitudes too large to be observed on real faults), is only $1 / 3 \log \beta$, which is $\approx .28$. This may seem a small amount, but from the point of view of seismic hazard, where recurrence times scale with slip, this means a factor of two difference.

It was satisfying that best-fitting measured values of $H$ correspond with seismogenic depths. How do we understand measured values of $\beta$ ? As noted before, the measured parameter $\beta$ can be understood as a combination of two effects: the additional slip at seismogenic depths due to the effective $W$ being larger than $H$, and the additional moment below the seismogenic depth. From the scaling of the 
largest events, we get

$$
\beta \approx \frac{2 W}{H}\left(1+\left.\frac{\mathcal{M}_{z>H}}{\mathcal{M}_{z \leq H}}\right|_{A / H^{2} \gg 1}\right) .
$$

Some sense of the rough size of $\mathcal{M}_{z>H} / \mathcal{M}_{z \leq H}$ can be obtained from dynamic models. Shaw and Wesnousky [2008] found ratios around .5 for this ratio for the frictions they studied. Because it is the sum of this ratio plus unity which matters, we are relatively insensitive to the exact value, and thus can take the value from Shaw and Wesnousky [2008] to get a rough estimate for $W$. Then $W \approx \beta H /(2 * 1.5) \approx 35 \mathrm{~km}$. This, interestingly, corresponds roughly with typical values of the depth of the crust.

Magnitude area scaling relations are one of the key branchpoints in seismic hazard calculations, and gives one of the dominant uncertainties in hazard estimates [WGCEP, 2007]. Here, we have show that a physical hypothesis of constant stress drop across all magnitudes gives a scaling with a better fit to the whole range of the magnitude-area data. This work also raises additional questions. The question of whether slip penetrates coseismically deeply below the seismogenic depth remains an open question, and observations which can unambiguously constrain this would be extremely valuable. Revisiting the question of area measuremjents at moderate magnitude events is also called for, particularly with new precise locations of aftershock available [Waldhauser and Ellsworth, 2000]. Finally, if earthquake stress drops are constant across all magnitudes, as this work suggests, the question remains: Why?

\section{Acknowledgements}

This work was supported by NSF EAR03-37226 and by the Southern California Earthquake Center (SCEC). I thank Bill Ellsworth, Art McGarr, and Tom Hanks for their thoughtfull comments on the manuscript.

\section{References}

Akaike, H., A new look at the statistical model identification, IEEE Transactions on Automatic Control, AC19, 716, 1974.

Aki, K., Earthquake mechanism, Tectonophysics, 13, 423, 1972.

Bodin, P., and J. N. Brune, On the scaling of slip with rupture length for shallow strike-slip earthquakes: Quasistatic models and dynamic rupture propagation, Bull. Seismol. Soc. Am., 86, 12921299, 1996.

Das, S., Appropriate boundary-conditions for modeling very long earthquakes and physical consequences, Bull. Seismol. Soc. Am., 72, 1911, 1982.

Hanks, T. C., Earthquake stress-drops, ambient tectonic stresses, and the stresses that drive plates, Pure Appl. Geophys., 115, 441-458, 1977.

Hanks, T. C., and W. H. Bakun, A blinear source-scaling model for M-log A observations of continental earthquakes, Bull. Seismol. Soc. Am., 92, 1841, 2002.

Hanks, T. C., and W. H. Bakun, M-log A observations of recent large earthquakes, Bull. Seismol. Soc. Am., 98, 490, 2008. 
Hanks, T. C., and H. Kanamori, A moment magnitude scale, J. Geophys. Res., 84, 2348, 1979.

Henry, C., and S. Das, Aftershock zones of large shallow earthquakes: fault dimensions, aftershock area expansion and scaling relations, Geophys. J. Int., 147, 272, 2001.

King, G. L., and S. Wesnousky, Scaling of fault parameters for continental strike-slip earthquakes, Bull. Seismol. Soc. Am., 97, 1833, 2007.

Manighetti, I., M. Campillo, S. Bouley, and F. Cotton, Earthquake scaling, fault segmentation, and structural maturity, Earth Plan. Sci. Lett., 253, 429, 2007.

Nazareth, J. J., and E. Hauksson, The seismogenic thickness of the Southern California crust, Bull. Seismol. Soc. Am., 94, 940, 2004.

Pegler, G., and S. Das, Analysis of the relationship between seismic moment and fault length for large crustal strike-slip earthquakes between 1977-1992, Geophys. Res. Lett., 23, 905, 1996.

Romanowicz, B., Strike-slip earthquakes on quasi-vertical transcurrent fault: inferences for general scaling relations., Geophys. Res. Lett., 19, 481, 1992.

Romanowicz, B., Comment on 'A reappraisal of large earthquake scaling' by C. Scholz, Bull. Seismol. Soc. Am., 84, 1675, 1994.

Scholz, C. H., Scaling laws for large earthquakes: consequences for physical models, Bull. Seismol. Soc. Am., 72, 1-14, 1982.

Scholz, C. H., Reply to comments on 'A reappraisal of large earthquake scaling, Bull. Seismol. Soc. Am., 84, 1677-1678, 1994.

Shaw, B. E., and C. H. Scholz, Slip-length scaling in large earthquakes: Observations and theory and implications for earthquake physics, Geophys. Res. Lett., 28, 2995, 2001.

Shaw, B. E., and S. G. Wesnousky, Slip-length scaling in large earthquakes: The role of deep penetrating slip below the seismogenic layer, Bull. Seismol. Soc. Am., p. in press, 2008.

Waldhauser, F., and W. L. Ellsworth, A double-difference earthquake location algorithm: Method and application to the northern hayward fault, california, Bull. Seismol. Soc. Am., 90, 1353, 2000.

Wells, D. L., and K. J. Coppersmith, New empirical relationships among magnitude, rupture length, rutpure width, rupture area, and surface displacement, Bull. Seismol. Soc. Am., 84, 974, 1994.

WGCEP, Earthquake probabilities in the San Francisco Bay Region: 2002 to 2031, U.S. Geol. Surv. Open File Rep., 03-214, 2003.

WGCEP, Uniform California Earthquake Rupture Forecast, 2007. 


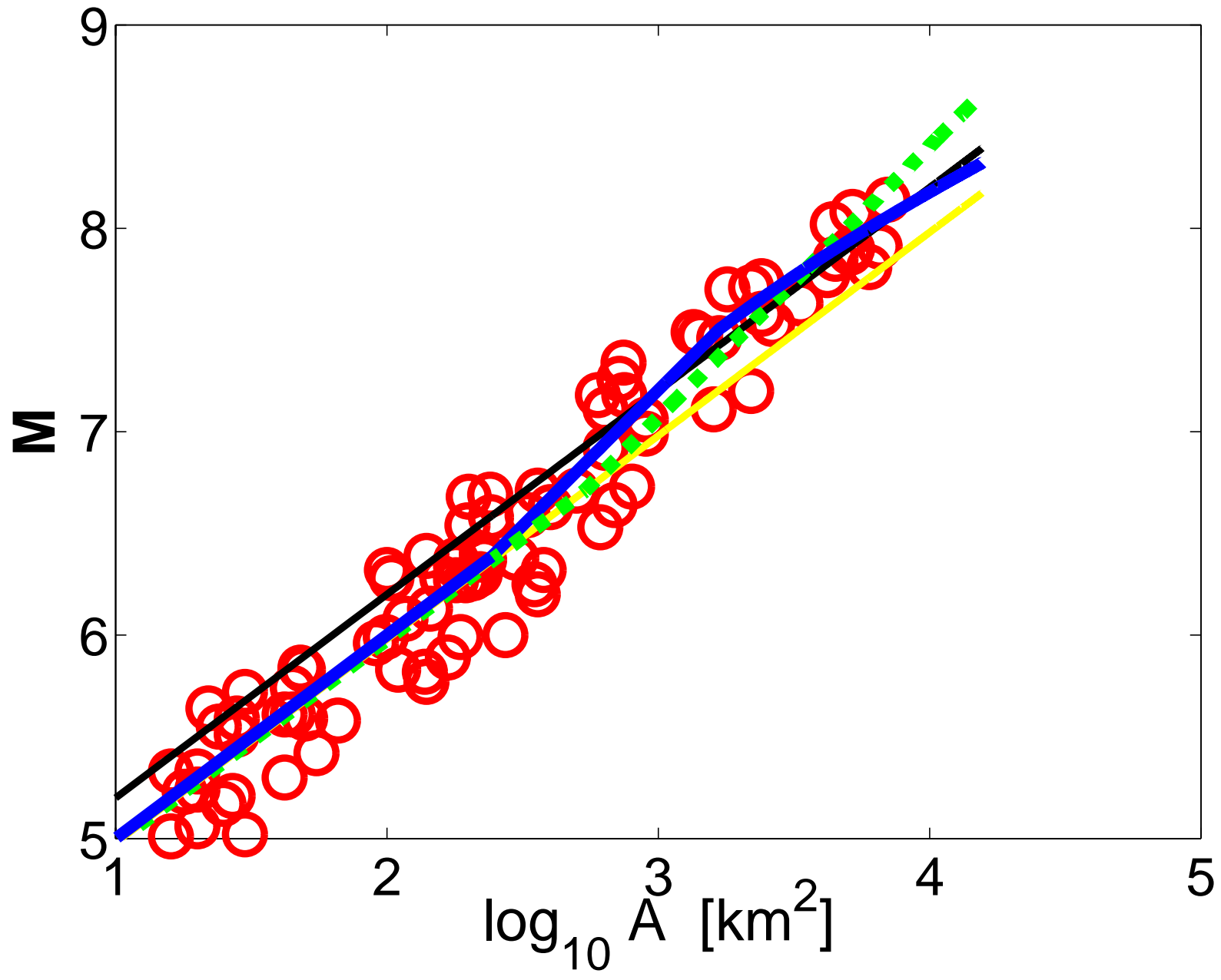

Figure 1: Magnitude area relations for large strike-slip events. Red circles denote magnitude and area of events from Hanks and Bakun [2008] database. Solid yellow line is linear Wells and Coppersmith [1994] mag-area relation, solid black line is linear Ellsworth-B [WGCEP, 2003] mag-area relation, Equation (1). Dashed green line is Hanks and Bakun [2002] bilinear relation, Equation (2). Blue line is our new proposed scaling relation, Equation (8), with least squares best fitting parameters $H=15.6 \mathrm{~km}, \beta=6.9$. Note excellent agreement of solid blue line with data across the whole range of magnitudes. 